\title{
Lugares cargados de memoria: aproximaciones hipotéticas sobre la construcción de identidad y territorio en los Tageiri y Taromenaneo
}

Kati Álvarez*

\begin{abstract}
RESUMEN
DENTRO DE LA CONSTRUCCIÓN DE LOS TERRITORIOS AMAZÓNICOS POR PARTE DE LOS GRUPOS AISLADOS, SE EXPLORAN LOS LUGARES DE LA MEMORIA DONDE PRÁCTICAS TEMPO ESPACIALES COMO EL RETORNO A SUS SITIOS DE ORIGEN, Y DENTRO DE ELLO, LAS FORMAS DE CONSUMO, DEFENSA Y CAPTURA QUE PERMITEN CONSTRUIR IDENTIDAD Y TERRITORIO; LA CREACIÓN VERNÁCULA DE LOS SITIOS ÚTILES, LA SIEMBRA DE BIODIVERSIDAD Y LA FORMACIÓN DE TIERRA PRETA; LAS CAPTURAS DE PARENTESCO E IDENTIDAD A TRAVÉS DEL MATAR, DEL HUIR, DEL MORIR Y DE LAS ALIANZAS MATRIMONIALES; Y, FINALMENTE, LA RESILIENCIA DE ESTOS GRUPOS EN TANTO SE APROPIAN DE NUEVOS SITIOS ÚTILES.

Si BIEN ES CIERTO QUE SE CUENTA CON POCA INFORMACIÓN SOBRE LOS TAGEIRI TAROMENANE, ESTE ARTí́culo LANZA ALGUNAS HIPÓTESIS SOBRE LOS LUGARES DE LA MEMORIA Y LA CONSTRUCCIÓN DE IDENTIDAD Y TERRITORIO. ESTAS HIPÓTESIS ESTÁN BASADAS EN ETNOGRAFÍAS FRAGMENTADAS PERO SOSTENIDAS Y LA EVIDENCIA PRESENTADA ESTÁ SUSTENTA EN PERSONAS DE LA NACIONALIDAD WAORANI DEL ECUADOR Y OTROS EXPERTOS.
\end{abstract}

Palabras Claves: TAgeiri - TARomenane - Lugares de LA MeMORIA - IDENTIDAD - TERRITORIO - RESILIENCIA.

\section{Abstract}

Within isolated groups' CONSTRUCTION OF AMAZON TERRITORIES, WE EXPLORE MEMORY-PLACES WHERE TIMESPACE PRACTICES SUCH AS RETURN TO PLACE-OF-ORIGIN PRACTICES WHEREIN CONSUMPTION, DEFENSE AND KIDNAPPING STRATEGIES ALLOW FOR THE CONSTRUCTION OF IDENTITY AND TERRITORY; THE VERNACULAR CREATION OF USEFUL PLACES, THE SOWING OF BIODIVERSITY AND THE FORMATION OF TERRA PRIETA; THE CAPTURE OF KIN AND IDENTITY THROUGH KILLING, ESCAPE, DEATH AND MARRIAGE ALLIANCES; AND FINALLY, THE RESILIENCE OF THESE GROUPS WHILE THEY ARE APPROPRIATING NEW USEFUL PLACES.

EVEN THOUGH THERE IS LITTLE INFORMATION ABOUT TAGEIRI-TAROMENE, THIS ARTICLE PRESENTS SOME HYPOTHESES ABOUT MEMORY-PLACES, IDENTITY CONSTRUCTION AND TERRITORY. THESE HYPOTHESES ARE BASED ON FRAGMENTED

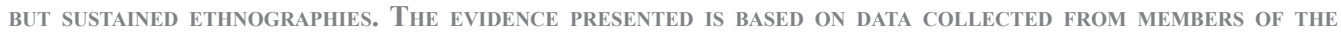
WaOrani Nation in ECUAdor AND OTHER EXPERTS

KEYWORDS: TAGEIRI - TAROMENANE - MEMORY-PLACES - IDENTITY - TERRITORY - RESILIENCE.

\footnotetext{
* Socióloga. Tiene una maestría en Ciencias Sociales con mención en Antropología. Actualmente cursa el doctorado en Historia Andina. Trabaja en la Amazonia ecuatoriana desde 2001 hasta la presente fecha. Se desempeña como docente en la Universidad Central del Ecuador. Correo electrónico: yoekam@gmail.com.
} 


\section{Introducción}

$\mathrm{V}$ olver al lugar donde uno o una ha nacido parece ser una práctica propia de algunos grupos amazónicos. El retorno espacio a los sitios de origen conllevan a una ciclicidad controlada socialmente. Esta ciclicidad implica una permanente construcción y reconstrucción de la memoria. Los lugares de los que se ha partido, y a los que se vuelve, contienen en sí formas de producción y reproducción de consumo, defensa y capturas. Estos elementos constituyen un conjunto histórico bastante complejo en el cual la ancestralidad está ligada a la relación sistemática entre cultura, utilidad e historia. En este sentido, el pasado tempo espacial es colocado como punto de referencia inicial y último de las prácticas sociales amazónicas.

Retomar los ríos, los senderos, seguir los pasos de los familiares muertos, escuchar sonidos conocidos, recolectar y sembrar, capturar y defender, volver a degustar y a oler lo vivido en la infancia, son agencias que construyen territorialidad y ancestralidad. Si bien varios antropólogos y antropólogas amazonistas sostienen que en la región amazónica no existen nociones de ancestralidad en muchas de las culturas, quizá, la ciclicidad histórica sea precisamente la construcción continua y sistemática de ancestralidad, y de lo que es un territorio ancestral.

El volver al lugar donde uno ha nacido supone una serie de eventos e inscripciones, una serie de hechos épicos, de lugares simbólicos rituales, de utilidad de espacios (cacería, pesca, horticultura, recolección), de estacionalidad en cuanto al acceso a los recursos. De alianzas matrimoniales o alianzas por muerte. De definición de propiedad y otras tantas determinaciones e inscripciones socio espaciales. Pero ¿qué ocurre cuando en este retorno a los lugares de origen y a los lugares de la memoria, algunos grupos amazónicos se encuentran con que ya no existen, con que llegó el olvido, o con que estos lugares ya no contienen lo creado y construido por ellos mismos en un presente y pasado incesante?

La resiliencia, o aquella capacidad que tienen las personas para afrontar las cosas nuevas, da cuenta de la utilización y re significación de estos nuevos sitios útiles.

Es importante mencionar que no existen muchos estudios sobre los Tageiri Taromenane. Pero, a pesar de ello, este artículo plantea, de manera hipotética, la construcción de lugares de la memoria, identidad y territorio que tendrían los grupos Tageiri, Taromenane y otras familias no contactadas, y la utilización / construcción por parte de estos grupos de los nuevos sitios útiles encontrados dentro de su territorio. Cabe señalar que no existe evidencia científica al respecto, pero para realizar estas reflexiones sobre los grupos aislados, se cuenta con estudios etnográficos fragmentados pero sostenidos desde el 2001 hasta 2016. Se han aplicado entrevistas, historias de vida y encuestas. Además de importantes referencias bibliográficas y documentales.

En este sentido, se inicia con una breve explicación de carácter teórico donde se puntualiza desde la geografía humana y política el enfoque del perspectivismo amazónico en cuanto a la construcción de lugares de la memoria, identidad y territorio en la cuenca amazónica, y se aplica similar enfoque para los grupos aislados. Posteriormente, se revisa la historia de autodeterminación de aislamiento de los grupos Tageiri, Taromenane y otras familias no contactadas.

Por otro lado, se plantean varios criterios sobre la creación de lugares de la memoria a través de la ciclicidad histórica y de las inscripciones territoriales de las formas de consumo, defensa y captura.

Finalmente, se hace mención a que en el proceso de construcción de los lugares de la memoria por parte de los Tageiri, Taromenane, y otras familias no contactadas, la presencia de actores externos, y de waorani contactados, han incidido en la capacidad que tienen estos grupos para mantener, rehacer, y re fabricar los rasgos característicos de consumo, defensa y captura. 


\section{Concepciones sobre territorios en la Amazonía}

Uno de los posicionamientos teóricos para revisar las concepciones sobre territorio en la Amazonia proviene del entendimiento del territorio como construcción socio cultural. En efecto, la escuela francesa de geografía conocida como el posibilismo, considera a los territorios como un elemento más de la acción social, ya que a su criterio, es el sujeto el que los determina o modifica (Febvre, 1961). Yi Fu Tuan en su trabajo "Humanistic Geography" (1976), reconoce a los sujetos como protagonistas y actores centrales de los lugares. Y en este sentido, los sitios son entendidos como la posibilidad de existencia, de habitar y de significación de lugares (Moreno Lache et al., 2013: 143).

En la amazonia, la acción social que construye territorios y hacen territorialidad depende de la perspectiva con la que las distintas culturas se apropian del entorno (en su patrón más tradicional). Por perspectivas o perspectivismo amazónico se entiende a un complejo entramado epistemológico, donde el pensamiento pan amazónico da cuenta de una serie de saberes, conocimientos y practicas propios de las culturas que allí habitan (Viveiros de Castro, 1996; Pazos, 2007).

En este sentido afirma Calavia (2004: 7), las concepciones territoriales en la amazonia tienen que ver con que "las relaciones sociales son en esencia relaciones espaciales. Y según Phillipe Descola (1996: 115), en particular, las sociedades guerreras se caracterizan por incorporar a través de la captura: personas, identidades, cuerpos y sustancias.

Sin duda, a esta composición del territorio, se suma lo mencionado por Viveiros de Castro (2009: 51), y esto es la cosmovisión antropomorfa que muchas culturas amazónicas tienen del medio físico, que a más de crear afectos y complicidades con el entorno considera a los seres no humanos (plantas y animales) con varios tipos de niveles de conciencia.

Al parecer, varios grupos amazónicos, entre ellos, la nacionalidad Waorani del Ecuador, así como probablemente, los grupos no contactados tanto de filiación lingüística waoterero/waotiriro como otras posibles; dejan ver en su acción social y en las relaciones sociales que mantienen entre sí y con el entorno, que aquello conocido como su territorio contiene la perspectiva del retorno a sus lugares de origen o ciclicidad histórica. Y que este conjunto histórico tempo espacial se nutre de formas de consumo, defensa y captura.

Al menos esto sugiere, por un lado la concentración en la actualidad de algunos ancianos y ancianas waorani en lo que se conoce como la antigua Guinta, sitio ubicado en la provincia de Orellana, cantones de Orellana y Aguarico, y el deseo de otros ancianos waorani por retornar al lugar donde nacieron para morir allí. Y por otro, el sin número de etnónimos encontrados en el territorio étnico waorani, en la zona intangible Tageiri, Taromenane, en el Parque Nacional Yasuní y dentro de bloques petroleros.

Como se observa en el MAPA 1, los nombres de algunas comunidades waorani, los nombres de ríos, e incluso los nombres de determinados recursos, hacen referencia a estos lugares como producto de su acción social, que sin duda, nutren y son el fundamento del concepto y sobre todo de la vivencia de lo que significa su territorio tanto para los waorani, como para grupos aislados Tageiri, Taromenane y otras familias no contactadas.

Solo por mencionar algo, la comunidad Quehueriono significa río de caníbales, la comunidad de Dícaro significa piedras, la comunidad de Baameno significa huesos; la comunidad de Timpoka significa un fruto muy parecido a la granadilla, o la comunidad de Kaaymeno que significa la comunidad de Kai, un importante guerrero (Onkiyenani Tenedadipa, 2009).

Es por esto que, la recurrencia al perspectivismo amazónico permite comprender los campos de relaciones socio espaciales e históricas con las que se producen territorios y territorialidad. Según Pazos (2007: 373- 375), a los territorios hay que pensarlos habitados, existen formas afectivas y formas de vida en ellos. Y, Laura Rival señala que "a los territorios hay que verlos como experiencia encarnada y material” (en Ibíd.: 375). 


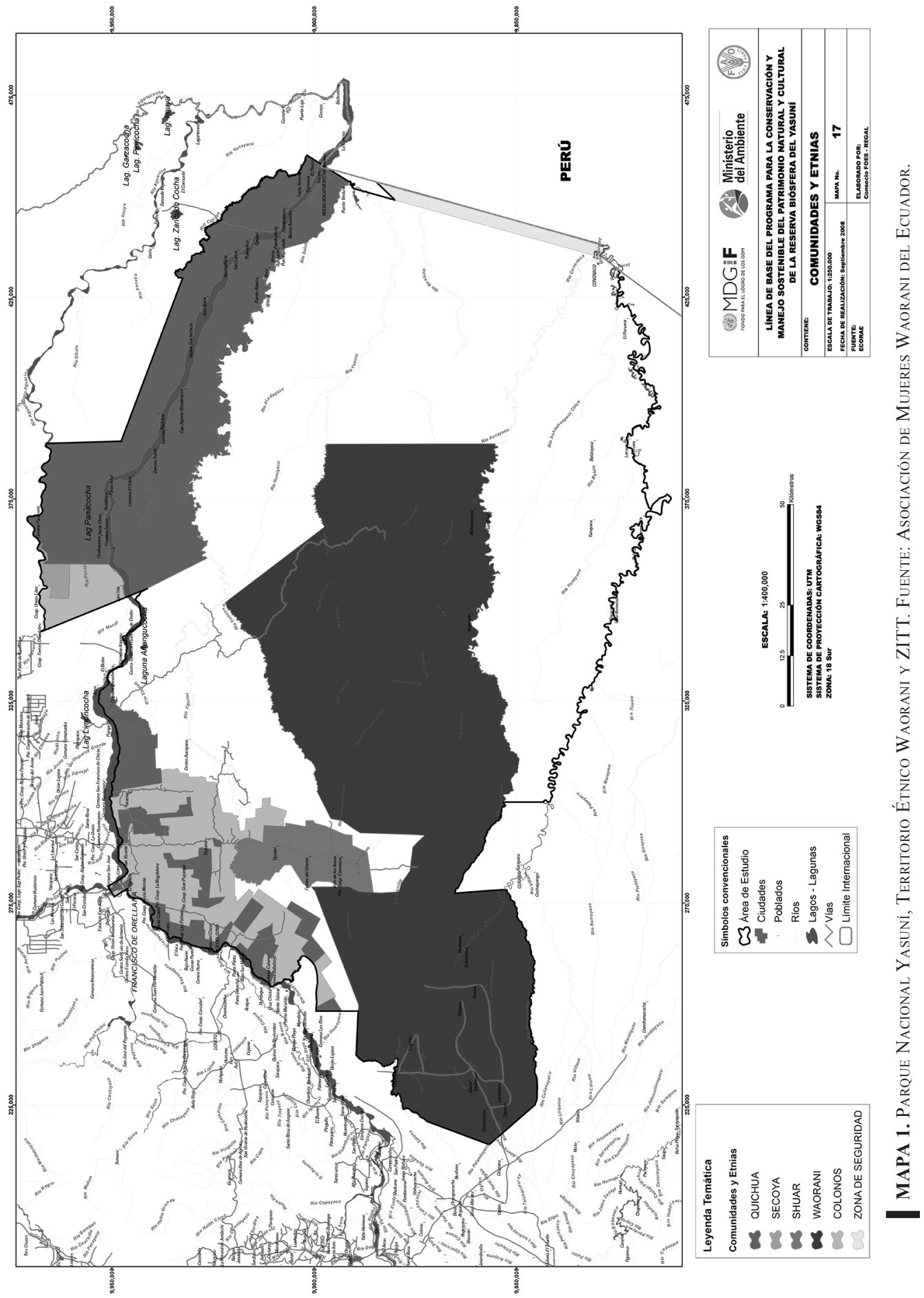


Desde este marco teórico así como desde las etnografías fragmentadas pero sostenidas es que se parte para proponer que los Tageiri, Taromenane y otras familias no contactadas construyen territorio y territorialidad desde la apropiación vernácula de los lugares. Es decir, que este conjunto histórico tempo espacial, que invita a regresar o retornar a los sitios de origen para vivir o morir allí, se nutre de formas de consumo, defensa y captura propios de grupos amazónicos como los waorani y otros más. Estas formas de consumo, defensa y captura implican formas de vida, experiencias encarnadas, materiales y simbólicas. Pero, además estas experiencias territoriales son ajustadas permanentemente dentro de las capacidades de resiliencia de las culturas para afrontar situaciones nuevas.

\section{Los Tageiri, Taromenane y otras familias no contactadas}

Aproximadamente por los años 50s se mencionaba la existencia de cuatro grupos waorani en lo que actualmente se conocen como las provincias de Orellana, Napo y Pastaza: los Guiketairi, Piyemoiri, Baiwieri y Wepeiri quienes ocupaban extensas áreas interpluviales para su residencia y sobrevivencia. De hecho, tres de estos grupos residían en el bajo río (Piyemoiri, Baiwieri y Wepeiri), aunque Colleoni y Proaño (2010) señalan que además en aquella época se contaba también con la presencia del grupo Taromenane.

Como se sabe, desde finales del siglo XIX hasta mediados del siglo XX, esta área ocupada por diversos grupos -entre ellos los Taromenane- fue el escenario de incursiones externas de diverso tipo: caucheros, hacendados, comerciantes de indígenas, militares ecuatorianos y peruanos. Además de aventureros, geólogos e investigadores pertenecientes a las compañías petroleras Leonard Company y Shell, y colonos (Cabodevilla, 2010). Al parecer, por estas presiones, los Taromenane deciden por su seguridad física internarse en la cabecera de los ríos Yasuní y Tiputini, y según Miguel Ângel Cabodevilla, se conoce que su líder fue Taromenga o Tarome (Cabodevilla, Smith y Rivas, 2004: 114).

El territorio ancestral Taromenane es conocido como Nemontare (Proaño, 2010). Esta versión coincide con lo expuesto por miembros de la nacionalidad waorani al afirmar que los Taromenane residían en Nemontare Guenwa (Álvarez y Boyotai, 2012). Nemontare Guenwa es el área comprendida entre los ríos Napo al norte (Ecuador) y Tigre al sur (Pastaza - Ecuador y Loreto- Perú).

Existen algunas posiciones que afirman que los Taromenane sostendrían cierta familiaridad con los waorani (Cabodevilla, 1999; Rivas y Lara, 2001). Incluso, algunos miembros de la nacionalidad waorani afirman que los Taromenane hablarían waoterero o waotiriro pero con acento distinto. Además, por el uso similar que se dan a las lanzas, y por algunos mitos de origen. De hecho, dentro de los mitos waorani se cree que los Taromenane se metamorfosearon en guanganas y se fueron a vivir en zonas interpluviales para evitar enfrentamientos con otras familias (Gondecki y Nenquimo, 2009).

Es por esto que para algunos waorani, Taromenane es un locativo que identifica a las personas que aún viven al interior de la selva. Incluso, algunas personas waorani sostienen tener lazos de parentesco con los Taromenane como Wiñeycamo de Kaiweimeno y Omatuki de Bataboro. (Álvarez y Boyotai, 2012).

Sin embargo, otros criterios confirmarían que los Taromenane tendrían otra filiación lingüística, quizá zapaorana. Esta última versión la confirma Tepaa Nenquimo, hermana de Tage en una entrevista realizada en 2012. Quienes sostienen esta hipótesis, comentan que existen algunas diferencias con los waorani: no se observan orejas perforadas, la elaboración de las lanzas y cerbatanas son distintas, y señala Izquierdo (1999) de las casas encontradas, éstas son más sólidas en comparación con las casas tradicionales de los waorani y cuentan con mamparas internas, cosa que no tienen las casas waorani.

En todo caso, lo que es indiscutible, es que los waorani reconocen en los Taromenane relaciones de afinidad socio cultural: excelentes y hábiles cazadores, guerreros y personas fuertes (Álvarez y Boyotai, 2012). 
Posteriormente, esto es por los años 70s, un grupo conocido como "los patas rojas" de filiación lingüística waoterero/waotiriro debido a presiones ejercidas sobre su territorio, - ya que se habían descubierto los campos Auca, Cononaco y Tiguino, y se planificaba el trazado de la vía Auca- ataca la ciudad del Coca (Cabodevilla, 2007). Dentro de los líderes que comandaron el ataque se encontraba Ñihua (Álvarez y Boyotai, 2012: 34). Al parecer, formaban parte de este grupo conocido en aquel entonces como "los patas rojas" miembros de los grupos Peyemoiri y Baihuairi.

Tras la muerte de Nihua que -según los waorani ocurrió por una grave enfermedad-, y la muerte de su hermano Kimontare en manos de su misma familia, algunos miembros del grupo optan por internarse en la selva y deciden no mantener contacto con agentes externos a los waorani; ni con otros waorani de sus mismos grupos familiares (Ibíd.: 47-48).

Este nuevo grupo pasa a ser comandado por Tage, hijo de Nihua, y es desde este momento en el que se presencia la decisión autónoma de un grupo de miembros waorani de aislarse. Para ello, y por las múltiples presiones y acontecimientos descritos, el nuevo grupo baja desde el alto Tiputini hacia los sectores de Shiripuno, Tigüino y Cononaco.

Es por esto que, el territorio de los Tageiri vendría a ser desde el alto Tiputini hasta los ríos Shiripuno, Tigüino y Cononaco. Esta área les fue heredada o son sus lugares de la memoria por sus grupos constituyentes: Peyemoiri y Baihuairi.

El sector donde deciden establecerse los Tageiri no es del todo desconocido para Tage y su grupo, ya que entre sus miembros existían descendientes Baihuairi y Peyemoiri y lo que hicieron fue retornar a sus lugares de origen. De hecho, el mismo Tage transitó por estos lugares cuando era más joven. En todo caso, para cuando el nuevo grupo retorna a estas zonas se encuentran con un área despejada.

Efectivamente, por los años 60s, el Instituto Lingüístico de Verano había trasladado al menos 100 Peyemoiri y otro tanto de personas del grupo Baihuari al Protectorado en Tihueno (Ibíd.: 48). Para ese entonces afirma Laura Rival (1996: 15) residían en Tihueno cinco sextos de la población waorani hasta el momento calculada. Es decir, el $90 \%$ de waorani residían en el área protegida (Ibíd.: 40). El 10\% restante no quiso acudir al llamado hecho por el ILV (se incluye al grupo de Tage).

Ahora bien, ocupados estos lugares por el nuevo grupo de Tage ocurre otro suceso. Por recomendaciones de James Yost, el ILV decide dispersar a los grupos que se encontraban en el protectorado (Ibíd.). Uno de estos grupos era el del líder Babe. Babe era miembro del grupo de los Baihuairi (enemigo de los Tageiri) y luego de que Babe perdiera a su hijo en un accidente, decide movilizarse junto con su grupo a territorio Tageiri, fundando la comunidad de Tiguino. Otras versiones sostienen que Babe decide moverse a esta área por el avance de las operaciones hidrocarburíferas (Petro Canada sería quien reubica a Babe) y esto se hizo con el objeto de frenar la colonización que se venía por la apertura de la vía Auca (colonos, kichwa, shuar). (Álvarez y Boyotai, 2012: 48).

Como ya se mencionó, los Tageiri mantenían y mantienen relaciones tensas con otros grupos waorani contactados -a más de los Babeiri-. De hecho un líder de Baameno describe que el grupo Cononaco y Gabaron tenía enfrentamientos con los Tageiri por venganzas, por muerte y para recuperar una mujer robada por ellos en los años 70s. Y como respuesta a estas incursiones por parte de los waorani, los Tageiri atacaron y mataron a Ampure y se distanciaron aún más de estos grupos (Cabodevilla, 2004: 56-57).

Con respecto a la presencia de otras familias waorani no contactadas, se presume que el grupo que reside en lo que se conoce como los bloques 17 y 14 son los Iwene o gente de los cocos, y al parecer tendrían relaciones de parentesco con los Taromenane (Proaño, 2010). Pero, y según el líder de la comunidad de Dícaro, existen también otras familias waorani no contactadas en la cuenca del río Nashiño. Este último dato coincide con avistamientos realizados en el Perú en lo que actualmente es la reserva de las cuencas de Napo y Tigre. Se desconoce qué líderes los comandan, por esta razón no se sabe a ciencia cierta la denominación de estos grupos o familias. 
Sin embargo, Lou registra waorani no contactados en las cabeceras del Napo y Tigre, y según moradores de los asentamientos en la frontera con el Perú, y la consultora GEMA (2007) se identifican los grupos Tageiri y Feromenani (Lou, 2003 y GEMA, 2007).

Hacia el sur y del lado del Perú, muy cerca en la frontera con Ecuador también se registran sub grupos Sapara y Abijiras, esto es en las cuencas de los ríos Curaray y Alto Napo. En lengua arabela se los denomina Pananujuri y de acuerdo a la descripción de las personas de las localidades cercanas, estas personas caminan desnudas y pintadas de achiote. Llama la atención la referencia a estos grupos como gente del oeste, es decir de la gente que vive en la puesta del sol. Para AIDESEP, en la misma zona existe otro grupo no identificado (Huertas, 2010).

\section{Grupos no contactados en el Ecuador}

Según estudios realizados por el CICAME existirían los siguientes grupos no contactados o en contacto inicial en el lado ecuatoriano: Grupo Cuchiyaku-Curaray, localizado al sur del río Shiripuno y sería el grupo sobreviviente de la matanza de 2003. Posiblemente mantendría contactos con las comunidades waorani de Baameno y Boanamo. La mayor parte de su segmento territorial estaría dentro de la Zona Intangible Tagaeri-Taromenani (ZITT).

Un segundo grupo, es el denominado Armadillo-Cononaco-Chico. Su emplazamiento ocuparía la zona más occidental de los territorios de estos grupos y estaría en la frontera de la colonización y las actividades petroleras. La proporción más grande de su segmento territorial está fuera de la ZITT, incluiría parte del territorio waorani y el Parque Nacional Yasuní (PNY). Además del territorio de comunidades colonas y waorani que están fuera de aquellas áreas. Este grupo estaría formado por los sobrevivientes de los Tageiri y quizá por un grupo conocido como Iwene, este grupo producto de la asimilación Tageiri-Taromenane.

El grupo Yasuni-Gabarón, localizados en la zona más oriental de los territorios de clanes aislados, entre el bajo Nashiño y el bajo Cononaco. Mantendría contactos con las comunidades waodani de Kawymeno y Baameno. Este podría ser el grupo del que hay indicios de presencia en la zona sur de los bloques petroleros 16, 31 e ITT. (VER MAPA 2)

Aunque existe otra hipótesis que habla de que en realidad podría tratarse de otros grupos familiares formados por waorani separados de sus antiguos clanes, que se mantienen en situación de aislamiento y que son quienes circulan por la frontera entre Ecuador y Perú. A continuación un mapa con las zonas de asentamientos de los Tageiri-Taromenane.

Al respecto, cabe señalar que según los waorani las personas no contactadas son un solo grupo de waorani conocido como Ñañewenani. Este grupo tiene varias familias filiales que lo que hacen es patrullar su territorio; o lo que queda de él. Al conocer que el área de mayor presión y amenaza a los Nañewenani es la de la vía Auca en los sectores de Pindo, Tigüino, Armadillo y Batabodo su defensa y ataque se concentran en esto sitios (Álvarez y Boyotai, 2012: 53).

\section{Los lugares cargados de memoria y la ciclicidad histórica}

Los lugares cargados de memoria en la Amazonia son el resultado de la ciclicidad histórica. Sin duda, la ciclicidad histórica es una práctica epistemológica que junta tiempo y espacio. Es un conjunto histórico que permite volver al origen. Esta vuelta al origen registrada en las personas adultas mayores de muchos grupos amazónicos, y de manera especial en la nacionalidad Waorani, y se presume que también en los grupos Tageiri Taromenane y otras familias no contactadas, facilita la continuidad material de los grupos a través de formas de consumo, defensa y captura, así como la continuidad simbólica e histórica de estos grupos a través de la memoria. Todas estas formas vernáculas de hacer territorio son inscritas en el mismo por los individuos de los grupos, y de allí deviene el carácter endogámico de territorialidad cuya utilidad es propiedad suya y de sus descendientes. 


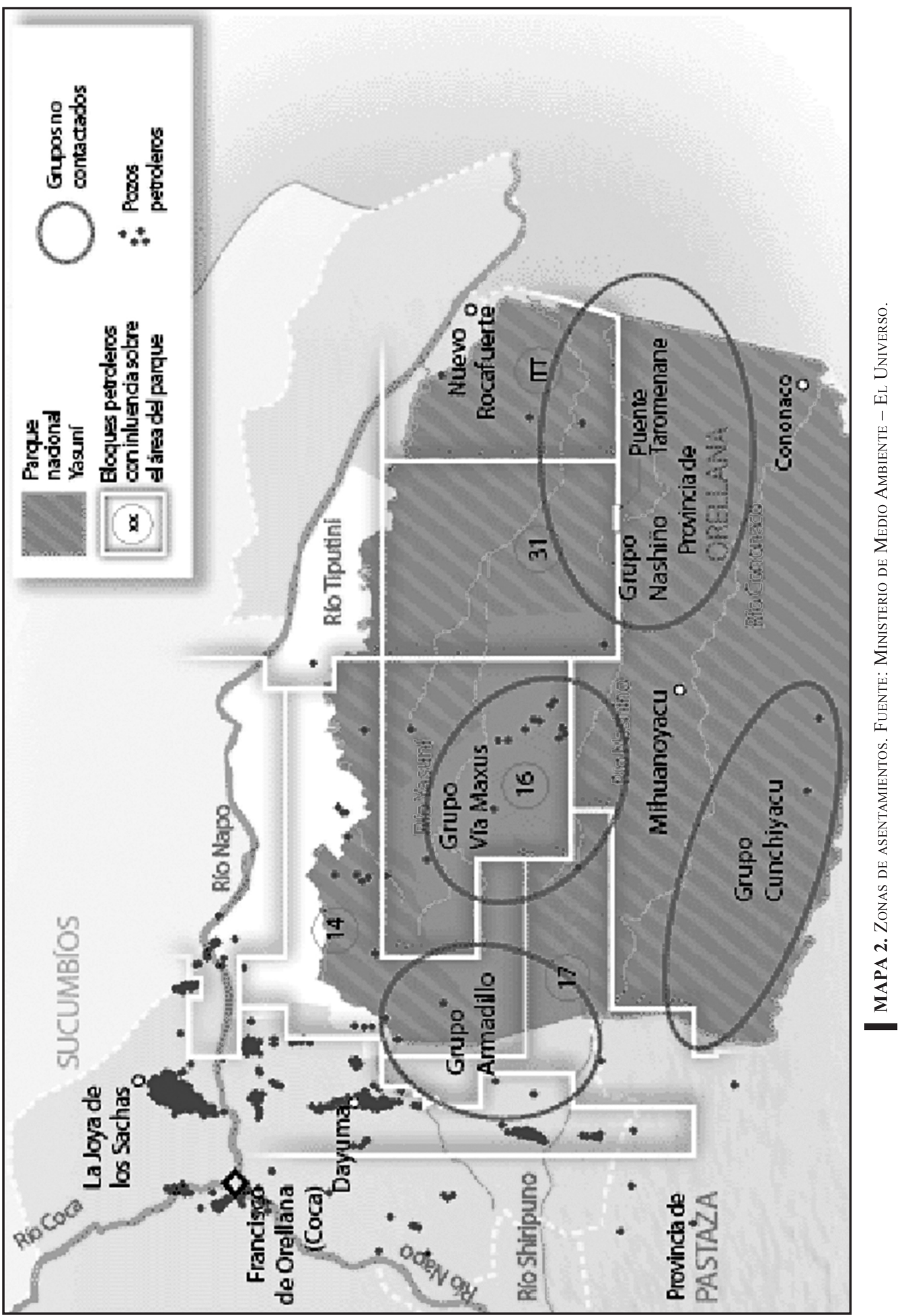


En este sentido, la utilidad del territorio es el resultado de un campo de relaciones sociales donde no existen anonimatos. Es decir, todo tiene su señal, inscripción o autoría. El territorio para los waorani, y se supone que para los grupos Tageiri Taromenane y otras familias no contactadas, es un campo de relaciones sociales que se extiende incluso a la capacidad relacional entre humanos y no humanos. Y precisamente, de estas construcciones es que sobreviene la ancestralidad. En este sentido, se puede afirmar que los territorios ancestrales son territorios que contienen utilidad y autoría. Son sitios útiles creados por los seres humanos, por lo tanto contienen cultura.

En la actualidad, se registra que casi la totalidad de los ancianos y las ancianas waorani han iniciado un éxodo que los lleva a retornar al lugar donde nacieron. No es sorpresa encontrar a varios ancianos y ancianas en la antigua Guinta, el sitio de residencia y movilidad de los grupos del bajo río. Mientras, otros ancianos o pekenani mencionan su deseo de regresar al alto Tivacuno, al Yasuní, al Nushiño, y otros sitios de origen para allí morir como es la tradición.

$\mathrm{Al}$ entrevistar sobre este tema del retorno a los abuelos y abuelas waorani se puede observar cómo sus miradas se pierden en el firmamento, y no siempre, pero a veces inician un canto que habla del vuelo de las aves, en especial, del águila harpía y los papagayos, y de la posibilidad de volver a esos lugares donde hubo buena comida, buena cacería y buena vida.

Sin embargo, muchos de estos ancianos afirman tener miedo de retornar a los sitios donde nacieron. Ahora, dicen algunos de estos lugares están habitados por los no contactados, quizá familiares, pero con los cuales existieron rupturas y conflictos que aún recuerdan. En tiempos no muy lejanos aseveran tuvieron enfrentamientos, y si vuelven, y si se encuentran con sus enemigos en este retorno, la memoria y la venganza llegarán. Otros ancianos mientras tanto, ven en sus sitios de origen muchos cambios: existen poblados, carreteras, colonos, petroleras. En estos sitios, algunas de sus inscripciones y autorías se mantienen, otras han desaparecido. Pero a pesar de ello, todos piensan regresar, sienten que es su deber hacerlo.

Si bien no se cuentan con similares testimonios del retorno a los sitios de origen por parte de los Tageiri Taromenane y familias filiales no contactadas se cuenta con registros de la movilidad trazada desde inicios del siglo XX y que dan cuenta investigadores como Miguel Ángel Cabodevilla. Al respecto cabe reflexionar sobre el por qué los Taromenane a inicios del siglo XX, presionados por las incursiones caucheras, resineras, hacendados, y otros; remontaron hacia las cabeceras de los ríos. ¿A qué se debió esta decisión?

Una posible respuesta sería porque a las cabeceras de los ríos no podían llegar tan fácilmente quienes hacían correrías, y estos sitios se convirtieron en refugios para algunas personas, al igual que las zonas interpluviales. Otra hipótesis, y que tienen que ver con la propuesta de los lugares cargados de memoria, sería que estos sitios fueron años anteriores sus lugares de residencia, de cacería, de horticultura o sitios de habitabilidad de los abuelos y abuelas (sean de filiación lingüística waoterero o zapaorana). O que quizá, ellos mismos vivieron y caminaron allí cuando fueron pequeños. De hecho, la movilidad de éste y otros grupos en la Amazonia está marcada por los sitios conocidos, los sitios trabajados, los sitios útiles.

Similar reflexión se puede hacer sobre la decisión del grupo de Tage que en los años 60s decide retornar al territorio de sus grupos fundadores: Peyemoiri y Baihuairi. Tage y su grupo deciden volver a sus territorios de origen y asentarse en los mismos. Es decir, bajan y se ubican por los ríos Cuchiyacu, Tiguino, Shiripuno. En este lugar, la memoria viva de sus abuelos y abuelas, y la propia está latente. En este lugar existen inscripciones y autorías que les indica que es su territorio. Sin dudarlo se puede afirmar que una de las inscripciones que indican el lugar de origen son las chontas. Así como los bosquecillos de yuca, de plátano, los sitios que huelen a maní o donde existen determinadas especies de animales.

Otras señales son los recuerdos de los épicos enfrentamientos entre grupos y familias, nacimientos, muertes. Sitios donde se recuerdan que son personas fuertes y sobre todo que son grandes caminantes. 
Estos elementos lanzados con el propósito de provocar discusiones sobre los lugares cargados de memoria, la construcción de identidad y territorio permiten entender la ciclicidad cumplida por estos grupos. De hecho, y por el registro que se tiene sobre su movilidad se puede comprender el por qué estos grupos de aislados -a pesar de encontrarse en un territorio ocupado por otros actores (colonos, petroleras, madereros, Estado, waorani enemigos)- deciden permanecer en él.

Mismos argumentos se pueden utilizar para señalar las dinámicas de movilidad que mantendrían otras familias filiales no contactadas. De hecho el grupo Nushiño-Gabarón, tendría desde esta perspectiva, cierta afinidad o lazos de parentesco con el grupo Wepeiri y se movería por esta razón por los ríos Yasuní, Ñushiño, Cononaco.

\section{Formas de consumo, defensa y captura}

A las formas de consumo, defensa y captura se las entiende como inscripciones o autorías espacio temporales y como elementos necesarios en la construcción de la memoria. En este sentido, es necesario recordar que la percepción que tienen algunos grupos amazónicos, y entre ellos los waorani y muy probablemente los grupos Tageiri Taromenane y otras familias no contactadas de su entorno, es que ellos hacen su territorio desde un caminar permanentemente cíclico, y no solamente estacional. Al respecto Calavia (2004: 7) señala: "Las acciones son desencadenadas por una partida o una llegada, los héroes matan, mueren o se transforman pero sobre todo, andan”.

\section{Producción y consumo}

Se ha dicho que la esfera del consumo organiza la vida social y económica waorani. Lo mismo podría decirse de los grupos Tageiri Taromenane y de otras familias no contactadas. Si bien la esfera del consumo afirma Laura Rival (2004) en los waorani es la que construye, mantiene y fortalece las relaciones sociales y espaciales, esto remite inmediatamente a la lógica económica registrada en estos grupos, y es que la producción debe ser individual y el consumo debe ser grupal endogámico.

Una manera de producir individualmente es caminar por la selva, o como algunas personas señalan, "ir de paseo". Cuando uno o una camina o va de paseo, va comiendo, sembrando, recolectando, registrando recursos, seleccionando y tratando semillas. Esta producción en gerundio de la selva se asemeja a un tipo de "polinización", que en el caso de las culturas amazónicas, es el resultado de un pensamiento y experimentación riguroso y milenario.

Esta polinización consiente se enriquece con un pacto social poco percibido por los antropólogos y es el que algunos grupos amazónicos siembran para humanos y no humanos. Sin embargo, es necesario reconocer las investigaciones de Roberto Pineda (1999) y Laura Rival (2004) y otros amazonistas que dan cuenta de esta complicidad de vida y relación con el entorno. Efectivamente, la producción individual de la selva es compartida con los humanos y con los no humanos. La lógica que atraviesa esta práctica, es que los no humanos, es decir los animales también siembran y comparten lo sembrado por ellos. Aquí se encuentra una diferencia con las propuestas de Descola en tanto relación con el entorno, y es la reciprocidad. Descola (2001) afirma que la reciprocidad con los animales es un principio de estricta equivalencia entre humanos y no humanos, donde los humanos devuelven a los no humanos la energía proporcionada por los alimentos.

En los waorani y quizá en los grupos no contactados, la reciprocidad está también basada en el criterio de antropomorfización de la naturaleza. Los animales -o los no humanos- realizan actividades o trabajos similares a los humanos. Los animales también producen la selva y comparten con los humanos lo producido. Esta lógica de complicidad se parece a lo que propone Calavia (2001: 161-176) y es que con los no humanos "existen afinidades en la cultura, la comida, en la habitabilidad e inclusive en el parentesco. Y además, existiría afinidad con los no humanos 
en la capacidad predatoria (Sifreddi, 2005:17 y Viveiros de Castro, 2002: 166). Entonces la reciprocidad en los waorani y muy probablemente en los grupos aislados vendría a darse desde este punto de vista, producir solo y consumir en grupo.

Dentro de esta relación social recíproca, los animales de cacería, especialmente las aves y los monos, también vendrían a ser criados en cierta medida por estos grupos. Es por esto que tanto los waorani como muy probablemente los Tageiri Taromenane y otras familias no contactadas utilizan el curare y otros compuestos vegetales para a través de todo un proceso de preparación y cantos convertir al animal en fruto. De ahí que cuando los cazan, no se refieren a ellos como piezas de cacería sino como piezas de recolección (entrevista de cacería, 2001, 2009 y 2013).

Por otra parte, dentro de los circuitos de retorno, tanto los waorani como quizá los grupos Tageiri Taromenane y otras familias no contactadas tienen claro las estacionalidades tanto de fructificaciónfloración, como de monos gordos y de algodón. De hecho, los registros estacionales que llevan estas personas son diarios y se los trasmite a través de los cantos y otras inscripciones en la selva:

"La estación de la chonta daguenca téré que viene desde enero hasta abril (y el sitio que se registra mayor número de chontas está próximo al río Shiripuno). La estación de los monos cebados yepenga téré que es desde junio hasta agosto (y el sitio con mayor número de monos gordos está hacia el Parque Nacional Yasuní y la ZITT; y la estación del algodón silvestre bohuéca téré que es desde septiembre hasta octubre y este producto es recolectado en cualquier lugar que tenga tierra seca" (Fundación Sinchi Sacha, 2005).

Mientras, la pesca no está exenta de esta relacionalidad y forma de producción -consumo, especialmente la pesca en riachuelos, arroyos y ríos pequeños. La pesca en los grandes ríos es una práctica relativamente reciente en los waorani y sin registros en los Tageiri Taromenane. Las áreas identificadas como buenas para pescar tanto para los waorani como para los aislados son los riachuelos próximos a la Zona Intangible Tageiri Taromenane y en el Parque Nacional Yasuní.

Estas prácticas de producción, del caminar y de los registros orales y espaciales van ligadas también a toda una lógica de reposición de suelos y recursos. Es decir, existe un estrecho lazo entre las culturas y la producción de la selva, de tierra preta y de la biodiversidad. Esto en conjunto viene a constituir para los waorani y probablemente para los grupos Tageiri Taromenane y otras familias no contactadas su entorno: crear sitios útiles de una manera vernácula para su sobrevivencia.

Añadir además que las dinámicas culturales tanto de los waorani como quizá de las personas no contactadas generan circuitos de recorrido estacionario y generacional que garantiza que en esa dinámica van a tener yuca, chonta, animales de cacería, y otros recursos que son recolectados de la selva. Los waorani tradicionales, y tal vez los grupos Tageiri Taromenane y otras familias no contactadas no hacen chacras en estricto sentido, sino kewenkode. Los Kewenkode vendrían a ser bosquecillos bio culturales donde se manejan plantas domesticadas y semi domesticadas, y donde se establecen lugares de cacería (Rival, 2004; Trujillo, 2008). Entonces, cuando se produce la yuca en uno o dos kewenkode, las personas waorani se movilizarán a una casa temporal hasta tener otra casa donde tendrán mayor permanencia. Así en los siguientes dos años, y de igual manera en las otras zonas de chonta, moretal y algodón. Se puede hablar de mínimo 12 -15 años de ciclos de movilidad por lo que la territorialidad o la noción de territorio tiene que ver también con el retorno o la ciclicidad histórica que construye sitios hortícolas útiles.

Tanto las chontas como las yucas y la recolección de otros productos de la selva, incluido los animales, o productos de las chacras -kewenkori- son inscripciones territoriales que guían el retorno a la tierra de los antepasados. Probablemente, estos mismos elementos son considerados por los Tageiri, los Taromenane y otras familias no contactadas para su retorno. En este sentido, se puede afirmar que la concepción de territorio para los grupos aislados pasaría por lugares cargados de memoria donde se visibilizan los trabajos realizados, las experiencias de habitabilidad, de ocupación, los aprendizajes recibidos por parte de los abuelos y abuelas de cada grupo en específico. 


\section{Formas de defensa y las capturas de parentesco e identidad}

Defender el territorio es defender la continuidad del grupo propietario del mismo. Pero también la defensa territorial forma parte de la identidad de estos grupos. Un guerrero waorani, y probablemente Tageiri o Taromenane, está llamado a defender su territorio y a su familia. Si no lo hace, su ser asume el papel de presa y deja de ser waorani (Álvarez, 2011). El ser persona en algunas culturas amazónicas pasa por la concepción predatoria y el ser presa desligitima la condición humana En este sentido, cualquier amenaza al territorio o a los recursos puede ser motivo de conflictos. Y cualquier amenaza a los miembros de la familia o el grupo (enfermedades, accidentes o muertes) es considerada como homicidio endogrupal y debe ejecutarse la venganza (Ibíd.).

Cuando los ataques, al menos en la nacionalidad waorani se concretan, se tienen dos opciones: el ataque o la huida. Tanto el ataque como la huida que impliquen o no muertes son marcadores territoriales. Estos marcadores territoriales definen como lo afirma Laura Rival las co-residencias; es decir, reconfigura la red de aliados y los enemigos en un territorio especifico. Al respecto, Oscar Calavia en relación a otros grupos amazónicos sostiene "que las marcas territoriales están inscritas por etnónimos, y éstos son lugares de la memoria que tienen relación con la captura, la huida o la guerra" (Calavia, 2004: 7 en Álvarez, 2011).

Los lugares cargados de memoria en los territorios de los waorani, y probablemente de los Tageiri Taromenane muestran los acontecimientos épicos ocurridos en relación a guerras o enfrentamientos con distintos grupos, incluso cuando estos enfrentamientos se han dado contra hacendados, colonos, petroleros, militares o madereros. Un claro ejemplo es el etnónimo Baameno, -que como ya se mencionó- significa "huesos", "la tierra de los huesos". Este nombre de una comunidad waorani fue elegido porque en ese sitio se dieron los enfrentamientos entre el ejército ecuatoriano y peruano en 1941. Además, y en simultáneo, se dieron enfrentamientos entre waorani y ambos ejércitos en el mismo período. En Baameno prácticamente existe un gran cementerio, de ahí su etnónimo.

Por otro lado, los sitios donde mayor conflictividad se ha registrado con los grupos Tageiri Taromenane ha sido precisamente donde mayor presión se ha ejercido por parte de petroleras, colonos y madereros. Esto es en el sector de la vía Auca, cerca de los campos Auca, Cononaco y Tigüino.

Haciendo un recuento histórico de ataques del grupo Tageiri-Taromenane en la zona cercana al campo Armadillo se tiene que en 1971 ocurre un enfrentamiento contra trabajadores de la compañía Western; luego en 1977 mueren lanceados tres trabajadores de la CGG, y muy próximo al río Shiripuno en 1987 muere monseñor Alejandro Labaka e Inés Arango. Este último acontecimiento ocurre un año después de que CEPE-TEXACO detecte al campo Armadillo dentro de territorio Tageiri. En el mismo año mueren cuatro personas más, esto incluye a dos personas Tageiri (Álvarez y Boyotai, 2012: 70).

En 1993 en el sector de Cuchiyaku fallece Carlos Omene y para 1993 el grupo de Babe secuestra a Omatuki (Cabodevilla et al., 2004). Posteriormente, en el 2002 mueren cinco madereros muy cerca de Ñoneno, en el 2003 mueren 16 personas del clan Tageiri-Taromenane. Como hechos recientes, entre el 2007 y 2008 se dieron varias muertes de madereros, entre ellos Mariano Castellanos; (Aguirre, 2007) y, en el 2009 mueren lanceados tres colonos en Los Reyes. Según Tepaa Nenquimo, hermana de Tage, el ataque a los colonos se debió a la intromisión de los mismos en territorio Tageiri Taromenane.

En el 2013 ocurre otro ataque, pero esta vez se da en la comunidad de Yarentaro, no en el sector de la vía Auca. Yarentaro se localiza en el cantón Aguarico de la provincia de Orellana. Los abuelos Ompure y Buganey mueren. No se sabe a ciencia cierta cuál de los grupos no contactados atacaron, y varias son las hipótesis sorteadas sobre las razones para que se haya dado el ataque. Lo que sí se puede afirmar, es que esta situación ocurre en este sector luego de algo más de 40 años desde el último enfrentamiento. 
Como se puede observar, los grupos Tageiri Taromenane y otras familias no contactadas hacen lo que los viejos guerreros waorani hicieron en su momento: defender su territorio y su familia para asegurar su continuidad, la de sus nietos, nietas; y de la defensa de la continuidad de la selva. Al parecer, la defensa territorial y grupal en su conjunto, permite sostener las relaciones espaciales, y las lógicas residenciales de estos grupos.

Pero, otra manera de capturar parentesco son las alianzas matrimoniales. A criterio de Tepaa Nenquimo, hermana de Tage, los grupos Tageiri, Taromenane y otras familias no contactadas seguirían buscando este tipo de alianzas principalmente con sus primos-as cruzadas. Esto, en su caso sería con sus hijos e hijas. Igualmente, ocurriría con las personas waorani que afirman tener parentesco con los grupos no contactados. Probablemente, el optar por primos cruzados para las alianzas matrimoniales tiene que ver con los troncos comunes parentales (abuelos y abuelas), y esto está muy ligado a la pertenencia espacial. Solo a través de esta vía de alianza es posible considerar a los territorios y recursos como propios.

Debido a los cambios ocurridos en cuanto a este punto, y por la reducida posibilidad de miembros para alianzas, los waorani creen que los Tageiri Taromenane y otras familias no contactadas no solamente consideran a primos cruzados para establecer alianzas sino a personas reconocidas por ellos como verdaderos waorani, -aunque sean enemigos-. Es decir, que al menos tengan y entiendan sus patrones de relacionamiento y que sean sus pares culturales. Esto último lo confirmó en una entrevista Ompure antes de su fallecimiento (Álvarez y Boyotai, 2012: 57).

Finalmente señalar que la captura de identidad en los waorani y posiblemente en los Tageiri Taromenane y otras familias no contactadas tiene que ver con ser y mostrarse como personas fuertes. Los grandes guerreros o las mujeres fuertes poseen un corazón exactamente igual de grande y predador que los jaguares, de manera especial como el wenonga meñe, una especie puntual de jaguar (Álvarez, 2011). Y esto como lo señala Surrallés, dentro de algunas culturas de las tierras bajas, es una condición de ser persona o poseer alma de persona (Surrallés, 2002/ 2003: 11-12).

Si bien a veces son necesarias las huidas para salvarse, cuando los individuos o grupos se sienten fuertes, regresan a vengarse. Es por esto que las huidas también son marcadores territoriales, ya que se procura borrar cualquier señal de haber sido o haberse sentido presa (Álvarez, 2011).

De los registros que se tienen sobre ataques y huidas de los grupos aislados se estima una concepción similar a la mencionada. Probablemente, un constitutivo de identidad como personas fuertes es la muestra de la fortaleza y bravura de los guerreros Tageiri Taromenane. Este reconocimiento a la fortaleza y bravura de los guerreros Tageiri Taromenane está registrado en las narraciones de varias personas waorani. Las referencias a la corporeidad de estos guerreros, a la velocidad con la que corren en la selva, y a la astucia para esconderse y ser invisibles ante los waorani dan cuenta de la profunda admiración y temor de las personas waorani a los grupos no contactados.

\section{Resiliencia de los Tageiri Taromenane y los nuevos sitios útiles}

Se había señalado que la resiliencia es la capacidad que tienen las personas para afrontar las cosas nuevas. Al respecto Thomas Mouriès señala lo siguiente:

"La creatividad adaptativa de los grupos amazónicos que: entre sus más notables recursos contra el riesgo de enajenación y disolución se encuentra su ingenio institucional y genio cosmológico para producir coherencia y continuidad - acaso a posteriori - en el proceso mismo de su transformación histórica" (Thomas Mouriès, 2014).

En términos geográficos y desde el perspectivismo amazónico, la resiliencia de los grupos amazónicos pasaría por considerar a los territorios ocupados con dinámicas no vernáculas y 
modernas, en muchos de los casos, como nuevos sitios útiles. Como bien lo dice Mouriés el ingenio institucional y genio cosmológico produce coherencia y continuidad dentro de las transformaciones históricas.

Estos nuevos sitios útiles de manera paralela están cargados de una memoria pasada y de una construcción presente del entorno. Al momento, en la región amazónica, coexisten otras experiencias territoriales y múltiples lugares de la memoria. Específicamente, dentro del territorio ancestral waorani constan algunas modalidades territoriales: Parque Nacional Yasuní, Zona Intangible Tageiri Taromenane y bloques petroleros (14, 17, 31, 16, ITT). Además de la construcción y el uso que distintas comunidades, centros poblados y empresas privadas (Lodges) hacen de los territorios y los recursos.

Dentro de este abanico de modalidades territoriales, los waorani y posiblemente los grupos aislados optaron por mantener su continuidad y prácticas de construcción territorial e identitarias. Sus lugares cargados de memoria han sido transformados, varios actores externos y ajenos a sus dinámicas lo habitan y lo re-significan.

De las pocas evidencias que se tiene, al parecer, los Tageiri Taromenane y otras familias no contactadas, han hecho uso de estos espacios como nuevos sitios útiles para su sobrevivencia. En el área donde se han registrado varios avistamientos de personas no contactadas, probablemente de los grupos Tageiri y Taromenane, se ha observado el patrón de consumo de los grupos en estado de guerra y con otras prácticas de apropiación del entorno. La recolección de alimentos no procesados como yuca, plátano, o de utensilios de cocina, baldes, machetes, plásticos o pedazos de telas es la tendencia.

Por lo general, a través de incursiones, como ha sido su lógica tradicional, estos grupos se han provisto de objetos de occidente y los han sumado a su cultura material. De hecho, los Tageiri Taromenane y otras familias no contactadas saben que estos objetos facilitan su reproducción social, y como muestra de ello se tienen algunos collares y lanzas donde se puede apreciar la incorporación de estos objetos occidentales a su cultura material. Lo mismo ocurre con los regalos recibidos por parte de los waorani. Cierto testimonio de un hombre probablemente Tageiri Taromenane en el sector de Pindo, provincia de Orellana confirma la incorporación de botellas de cola como recuerdos atesorados por los aislados, y como muestra de reciprocidad con ciertos miembros de la nacionalidad waorani (Álvarez y Boyotai, 2012: 71).

El consumo de estos artículos encontrados, recolectados o regalados da cuenta de la resiliencia que han desarrollado estos grupos, a la vez que se ha constituido en pocos casos como elementos de socialidad con algunas personas principalmente waorani.

\section{Conclusiones}

Lugares cargados de memoria: aproximaciones hipotéticas sobre la construcción de identidad y territorio en los grupos Tageiri Taromenane y otras familias no contactadas pasa por juntar lugar y tiempo. La práctica del retorno o la ciclicidad histórica producen formas de consumo, defensa y capturas necesarios para construir ancestralidad y por ende territorios ancestrales, pero sobre todo construyen memoria. La ancestralidad y la memoria desde esta perspectiva están ligadas a la relación sistemática entre cultura, utilidad e historia. En este sentido, el pasado y el presente tempo espacial es colocado como punto de referencia inicial y último de las prácticas sociales amazónicas.

El territorio está provisto de varias inscripciones o señales socio-espaciales que dejan ver la presencia de los ancestros: la misma selva, los familiares y los enemigos. La posibilidad de sobrevivir se transforma en una acción constante y consiente del caminar, del registrar lo hecho y lo que está por hacerse.

Si bien los grupos Tageiri Taromenane y otras familias no contactadas hacen uso de nuevos sitios considerados desde su perspectiva como útiles para la obtención de objetos occidentales, semi industrializados o industrializados, rondan varias interrogantes: ¿estamos los actores 
externos preparados para una convivencia pacífica con los grupos Tageiri, Taromenane y otras familias no contactadas?

¿Es posible flexibilizar las estructuras, las normas y los usos de los territorios considerados como hegemónicos?, y finalmente, ¿en qué medida se puede reconocer y valorar estas formas de construcción territorial vernáculas en las que los miembros de estas culturas son protagonistas de la altísima biodiversidad registrada en el área?

Los grupos Tageiri, Taromenane y otras familias no contactadas se encuentran en la actualidad muy vulnerables, especialmente porque sus formas de consumo, defensa y captura cada vez se han vuelto más inestables y dependientes de los actores externos. El regreso a los sitios de origen y la ciclicidad histórica de estos grupos están en peligro, su territorio, sus sitios útiles, sus sitios de la memoria y de la cultura están por desaparecer, y junto con ellos, un conjunto de saberes, conocimientos, lenguas y humanidades.

¿Por qué escribir sobre los grupos aislados?, ¿cuál es el interés personal y profesional por reflexionar acerca de estos grupos de personas que optaron por el aislamiento? Y ¿por qué escribir sobre los lugares cargados de memoria, identidad y territorio? A mi parecer, una de las razones para estas reflexiones, en su mayoría hipotéticas- es provocar discusiones y debates en el ámbito antropológico que pongan en juego mecanismos y herramientas amigables para comprender las dinámicas de grupos como los Tageiri Taromenane respetando su autodeterminación. Pero, sin duda, el interés principal para investigar sobre los Tageiri Taromenane viene del deseo y el deber ético de aportar - a través del conocimiento- con los derechos humanos y los derechos colectivos de estos grupos. Los Tageiri Taromenane y otras familias no contactadas requieren de su territorio, identidad y memoria para sobrevivir. Sin duda, creo que un mejor entendimiento de las distintas culturas, contactadas o no, generarán periodos de convivencia, tolerancia y paz.

\section{Bibliografía}

Aguirre, Milagros. 2007, iA quien le importan esas vidas! Un reportaje sobre la tala ilegal en el Parque Nacional Yasuní, Centro de Investigaciones Culturales de la Amazonía Ecuatoriana, CICAME, Quito.

Álvarez, Kati. 2011, Prácticas funerarias en los waodani, Flacso, Abya-Yala, Quito.

Álvarez, Kati y Rubén Boyotai. 2012, Investigación pueblos aislados. Situación del grupo Armadillo-Cononaco Chico-Vía Tigüino, Informe preparado para la Fundación Pachamama, Quito.

Belaunde, Luisa Elvira (Eds.) 2009, "De seres y perspectivas", en: Amazonía Peruana, Centro Amazónico de Antropología y Aplicación Práctica CAAAP, Tomo XV, № 30, Lima, pp. 1-55.

Cabodevilla, Miguel Ángel. 2010 La Nación Waorani: Noticias Históricas y Territorio, Vicariato Apostólico de Aguarico, Unión Europea, Orellana Ecuador.

2007, Coca. La región y sus historias, Vicariato Apostólico del Aguarico, Centro de Investigaciones Culturales de la Amazonía Ecuatoriana CICAME, Quito.

2004, Oro creciente, y otros relatos de selva adentro, Vicariato Apostólico del Aguarico, Centro de Investigaciones Culturales de la Amazonía Ecuatoriana CICAME, Quito.

1994, Los Huaoranis en la Historia de los Pueblos del Oriente, CICAME, Navarra España, Quito.

Cabodevilla, Miguel Ángel et al. 2004, Tiempos de guerra. Waorani contra Taromenane.: Abya-Yala, primera edición, Quito.

Calavia, Oscar. 2004, "Mapas Carnales, el territorio y la sociedad Yaminawa”, en: Surrallés, Alexandre y Pedro García Fierro (Eds.) Tierra Adentro: Territorio Indígena y Percepción del entorno 5, IWGIA, Copenhage, pp.121-130.

2001, "El rastro de los pecaríes: Variaciones míticas, variaciones cosmológicas e identidades étnicas en la etnología pano", en: Journal de la société des américanistes [En ligne], 87 / 2001, mis en ligne le 17 novembre 2005, vista el 22 de junio de 2010. URL : http://jsa.revues.org/index1846.html.

Colleoni, P. y J. Proaño. 2010, Caminantes de la Selva, Informe 7. IWGIA. 
Descola, Phillipe. 2001 "Construyendo naturalezas. Ecología simbólica y práctica social” En Naturaleza y Sociedad, Perspectivas Antropológicas. P. Descola y G. Pálsson (eds): 101-122. México. Siglo XXI.

Descola, Phillipe y Pálsson, Gísli. 1996, Nature and Society: Anthropological Perspectives, Routledge, Londres. Febvre, Lucien. 1961, La tierra y la evolución humana. Introducción geográfica a la Historia [1922], con la colaboración de Lionel Bataillon, $2^{\mathrm{a}}$ edición en español, Unión Tipográfica Editorial Hispano Americana, primera parte. México

Fundación Sinchi Sacha 2005, "Fundamentos culturales para la iconografía y simbología artesanal de la nacionalidad huaroni”. Fundación Sinchi Sacha, en: pdf.usaid.gov/pdf_docs/PNADF536.pdf

GEMA. 2007, Estudio de Impacto Ambiental. Sísmica 2D, Lote 39, Perú.

Gondecki, Philip y Nenquimo Ima. 2009, "Wenonga Meñe, el guerrero Jaguar. Tradición Oral y Patrimonio Cultural del Yasuní", en: Oralidad para el Rescate de la Tradición Oral de América Latina y el Caribe, Anuario 16, UNESCO, La Habana-Cuba, pp.6-13.

Holling, C. S. 1973, "Resilience and Stability of Ecological Systems", Annual Review of Ecology and Systematics, Vol. 4, pp.1-23.

Huertas, Beatriz. 2010, Análisis de Situación de los Pueblos en Aislamiento, Contacto Reciente y Contacto Inicial de la Región Andina. Organismo Andino de Salud - Convenio Hipólito UNANUE ORAS - CONHU.

Izquierdo, Juan. 1999, La eco arquitectura: asentamientos humanos waorani, Abya-Yala, Quito.

Lou, Sonia. 2003, Informe del viaje realizado al distrito de Napo, Informe de Viaje No 001-2003/DP-PCN. Defensoría del Pueblo, Lima.

Moreno Lache, Nubia et al. 2013, "Pensar e indagar la construcción social del espacio: balances y retos", en: Revista Folios, $\mathrm{N}^{\circ}$ 38, Universidad Nacional Pedagógica de Colombia, pp. 141- 156.

Mouriès, Thomas. 2014, “¿Con o sin ancestros? Vigencia de lo ancestral en la amazonia peruana”, en: Anthropologica vol. 32. $\mathrm{N}^{\mathrm{o}}$ 32, Lima, pp. 17-40.

Onkiyenani Tenedadipa. 2009, Las voces de las mujeres, Corporación Humanas Ecuador, Asociación de Mujeres Waodani del Ecuador, Ministerio de Cultura.

Pazos, Álvaro 2007, [Reseña] "Tierra adentro, territorio indígena y percepción del entorno", en: AIBR. Revista de Antropología Iberoamericana, mayo - agosto año / vol. 2, № 2, Antropólogos Iberoamericanos en Red, Madrid, España.

Pineda, Roberto. 1999, "Sembrando la selva", en: Colombia Maguaré, Universidad Nacional de Colombia. vl.1. Fascículo 14, Bogotá, pp. 264-283.

Proaño, José. 2010, Voces de la Selva. Noticias recientes sobre los pueblos indígenas aislados en la Amazonía Ecuatoriana, Fundación Alejandro Labaka.

Rival, Laura. 2004, "El crecimiento de las familias y de los árboles: la percepción del bosque en los Huaorani", en: Surrallésn Alexandre y Pedro García Fierro (Eds.) Tierra Adentro:Territorio Indígena y Percepción del entorno, IWGIA, Copenhage, pp. 97-120.

1996, Hijos del Sol, padres del jaguar: Los huaorani de ayer y de hoy, Abya- Yala, Quito.

Rivas, Alex y Rommel Lara. 2001, Conservación y Petróleo en la Amazonía Ecuatoriana. Un acercamiento al caso Huaorani, Eco-ciencia, Abya-Yala, Quito.

Siffredi, Alexandra. 2005, "Cuando la persona se deshumaniza: descentramiento y jaguarización en la sociedad nivaclé", en: Journal de la Societé des américanistes [En ligne] 91-1/ 2005, mis en ligne le 10 janvier 2010. [visto el 30 de enero 2010. URL] Disponible en : http://jsa.revues.org/index2902.html.

Surrallés, Alexandre. 2002/2003, "De la percepción en Antropología. Algunas reflexiones sobre la noción de persona desde los estudios amazónicos", en: INDIANA 19/20. (2002/2003), S 59 - 72. [Consulta el 11 Abr 2011 17:09:26 GMT] en: http://www.uni-muenster.de/Hispanistikbibliographie/Bibliographie\%2020022004/dateien/LS9.htm.

Tuan, Yi - Fu. 1976, Humanistic Geography, Ann. Assoc. Am. Geogs. 66, pp. 266-276.

Trujillo, Julio. 2008, Diagnóstico de situación socio económico y cultural de las comunidades Waorani y Kichwa del área de influencia de Repsol-YPF.

Viveiros de Castro, Eduardo. 2002, "Perspectivismo e multinaturalismo na América Indígena", en: A inconsistencia de alma salvagem e otros ensayos de antropología, Cosac \& Naify, Sao Pablo, pp. 347-399.

1996, “Os Pronomes Cosmologicos e o Perspectivismo Ameríndio”, en: Mana 2 (29), pp. 115-144. 